\title{
在建筑工程造价管理中 BIM 技术的运用探讨
}

\section{Discussion on the application of BIM Technology in construction cost management}

\author{
曹永香 \\ Yongxiang Cao \\ 志丹县财政预算评审中心 陕西 延安 717500 \\ Zhidan County budget evaluation center Yan an Shaanxi 717500
}

\begin{abstract}
摘 要: 建筑行业作为我国社会发展的重要组成部分,对于城市化进程有着非常重要的影响,建筑市场内部的竞争也会变得 越来越激烈。就从目前情况看来, 传统的建筑行业发展模式已经无法适应时代发展的需求, 为了能够让建筑企业得到进一步 发展, 企业不仅要对实际发现过程中存在的问题予以足够重视, 并且根据实际情况来采取措施进行处理,这样才可以达到良 好的效果。所以,建筑企业在工程造价管理过程当中要对 BIM 技术进行充分的分析,并且对该技术进行充分应用,这样才可以 达到良好的发展。
\end{abstract}

\begin{abstract}
China's social development, the construction industry has a very important impact on the process of urbanization, and the competition within the construction market will become more and more fierce. From the current situation, the traditional development mode of the construction industry has been unable to meet the needs of the development of the times. In order to further develop the construction enterprises, the enterprises should not only pay enough attention to the problems existing in the actual discovery process, but also take measures to deal with them according to the actual situation, so as to achieve good results. Therefore, in the process of project cost management, construction enterprises should fully analyze the BIM Technology, and fully apply the technology, so as to achieve good development.
\end{abstract}

关键词: 建筑企业; 工程造价管理; BIM 技术; 实际运用

Keywords : construction enterprises; project cost management; BIM Technology; practical application

DOI : $10.36012 /$ etr.v2i12.3069

通过实际调查发现，我国整体经济水平提高的同时,社 会各个领域的相关工作技术与工作结构也在不断的转变, 建 筑行业当中也诞生了各种各样的施工管理方式与施工工艺 新形式。为此, 建筑企业要对工程造价管理工作予以足够的 重视, 并且根据实际的情况来对 BIM 技术进行合理应用, 这 样不仅能够对建筑企业的发展起到促进作用, 而且还可以达 到预期城市化发展的效果。

\section{BIM 技术的特点}

\section{1 可视化}

在 BIM 技术当中有着较多的特征,其中最为重要的特征 之一就是可视化, 建筑企业可以通过 BIM 技术来将三维数 据建模功能充分发挥出来, 进而转变传统二维施工图纸当中 存在的弊端, 相关工作人员可以通过三维立体模型来对各个 阶段的施工环节进行充分的了解, 进而可以掌握各个环节当 中的相关信息和数据。在通常的情况下, 建筑功能和建筑构 造随着时代发展而变得越来越复杂, 如果施工单位还在沿用 传统的二维图纸, 那么就会导致实际施工会存在局限性, 建
筑施工造价工作无法正常开展。另外, BIM 技术不仅能够为 工作人员提供正确的可视化思路, 而且还可以在实际应用过 程中对各个方面的信息进行整合, 在设计图纸当中对重要内 容进行标注, 在此基础上建立可视化的数据信息模型。

\section{2 协调性}

在建筑工程造价管理过程中可以发挥出非常重要作用 的就是 BIM 技术的协调性, 在以往的施工过程中, 各个部门 的工作人员要进行相互协商与沟通, 在最后意见达成一致的 时候需要建设企业投入较多的人力、物力和财力, 这样才能 达到预期的工作效果, 协调工作可能无法发挥出其应有的作 用。除此外, 建筑企业通过 BIM 技术可以对施工过程中的协 调性问题进行有效处理, 在整个过程中达到良好的协调管理 效果, 工作人员在此基础上可以对三维建模功能进行充分应 用, 对相关数据信息进行充分的分析, 这样可以在分析过程 中对一些问题予以足够重视, 进而在第一时间采取有效措施 进行处理, 建筑工程的协调工作速度也会变得越来越快。

\section{2.传统建筑工程造价管理工作存在的问题}




\section{1 工程造价模式与市场脱节}

现如今, 我国社会体系随着社会理念的变化而得到了进 一步的变化, 为了能够让建筑企业可以对工程造价进行宏观 调控, 相关管理人员可以根据实际的情况来采取相应措施进 行合理的干涉。不过我国部分建筑企业在工程造价管理工作 当中将动态管理与静态管理模式进行充分结合, 尽管在实际 应用过程中取得了良好的效果, 不过不同地区发展水平有着 不同的差异,进而就会对整体的工程造价管理工作当中还会 存在计划特征, 最终就会导致建筑企业与市场发展趋势相违 背, 我国建筑行业的健康发展也会因此而受到影响。

\section{2 工程计价具有区域性}

在通常的情况下, 我国不同的地区有着各自的区域特 点, 而且在经济发展水平与民俗特征方面也有着较大的差 异, 为了能够让各个地区得到更好的发展, 建筑企业在开展 工程造价管理过程中要建立区域特色的发展趋势。但是, 我 国不同的行业在实际发展过程中形成了各自的行业特色,这 些行业特色对于相关企业的发展有着一定程度的影响,工作 人员在熟悉本区域及本行业造价管理模式的同时, 日常工作 过程中需要对大量的数据信息进行处理,这样就会导致造价 工作过程中出现失误, 严重的时候还会对造价工作带来影 响, 企业无法得到更好的发展。

\section{3 造价数据难以共享}

就从目前情况看来, 建筑企业工程造价管理工作正常开 展的重要前提就是对相关数据进行收集和整理, 不过在这个 过程中涉及到的造价数据种类比较多, 在确定造价过程中还 有部分企业向供应商寻求价格,最后将这些信息放到网上或 者印刷成册, 建筑企业应用这种造价管理方式不仅日常的工 作量比较大,而且管理人员无法对各个环节的信息数据进行 充分掌握。另外,工作人员在对信息整合完成之后无法进行 实时分享, 这样就会导致造价管理工作无法达到实时分享的 效果，进而建筑企业自身的发展也会受到一定程度的影响。

\section{BIM 技术在建筑工程造价管理中的运用 \\ 3.1 工程投资决策阶段中的应用}

建筑工程在实际开展施工之前需要进行工程投资的决 策，在这个阶段当中应用 BIM 技术可以将可视化与模拟性 的功能充分发挥出来, 相关管理人员可以对工程造价预算等 方面来进行科学的模拟, 并且与相似的工程进行相互比较。 另外, 建筑企业工作人员还要在这个过程中对工程量进行科 学的计算, 根据企业造价评估指标进行充分分析和了解, 进 而可以得到真实的尖嘴工程总体的投资概算状况，为工程制 定规范科学的决策起到一定的促进作用, 各个施工环节当中 有着较多的技术支持。

\section{2 建筑工程设计阶段中的 BIM 技术运用}

通过实际调查发现, 在建筑工程整体费用当中占据比例 较小的阶段就是工程设计阶段, 尽管这个阶段占据费用比例 比较小, 不过设计阶段在建筑工程造价管理当中占据着非常 重要的地位。所以, 建筑企业可以根据实际的情况来进行充 分分析, 在此基础上来将 $\mathrm{CAD}$ 图纸当中的各种要素与数据 造价信息进行充分的结合, 通过时间维度来对建设或部分工 程进行选择性的分析, 这样才可以在设计阶段呈现出各个环 节的造价信息, 进而达到预期的限额设计目的。在以往的图 纸会审与设计交底工作当中, 设计部门会将施工图作为平面 图纸, 在图纸设计过程中将各种元素进行有效的分离, 这样 才能够让工作人员对一些细节部分予以足够重视。除此之 外, 建筑企业通过 BIM 技术还可以将建筑结构、土建及安装 等方面进行整合, 并且还可以对立体化的数据信息进行处 理, 通过 $3 \mathrm{D} 、 4 \mathrm{D}$ 等方式来呈现, 工作人员可以对设计工作当 中存在的缺陷进行充分分析, 在第一时间采取措施进行处 理, 这样才可以进一步提高建筑工程整体的质量。

\section{3 建筑工程施工中的 BIM 技术运用}

建筑工程施工过程中最主要的特点就是施工周期比较 长, 建筑企业受到市场与频率波动带来的影响比较深, 进而 建筑工程项目造价管理工作整体的难度也比较高。建筑企业 对 BIM 技术进行运用可以将模拟性、协调性和可视性效果 充分发挥出来, 提前发现工程施工之前遗漏的设计疏漏, 这 样才可以避免后期出现返工和设计变更等问题。相关工作人 员还要不断提高自身的综合素质和专业知识水平, 对 BIM 技术进行充分的分析和了解, 严格按照相关的要求和规定来 对该技术进行充分应用, 这样不仅该技术的效果可以充分发 挥出来, 建筑工程造价管理整体的科学性和有效性也会得到 进一步提高。

\section{4. 结语}

综上所述, BIM 技术在建筑工程造价管理当中可以发挥 出非常重要的作用, 为了能够进一步提高工程造价管理工作 的效率和质量, 建筑企业要对该技术予以足够重视, 并且在 实际的发展过程中进行充分应用。另外, 工作人员也要将该 技术应用到各个造价管理环节当中, 这样可以对其中存在的 问题进行及时处理, 建筑企业可以在激烈的市场竞争当中站 稳脚跟, 市场竞争力提高的同时, 还可以获取一定的经济利 益, 工程造价管理工作能够达到预期的工作效果, 我国建筑 行业整体发展也会得到促进。

\section{参考文献}

[1] 倪点.BIM 技术在建筑工程造价管理中的应用探讨 [J]. 建材与 装饰,020,No.612(15):161+164. 